\title{
El Uso productivo del Tiempo Libre vivenciado desde la Animación Sociocultural con personas mayores
}

\author{
Monsalve Robayo , Angélica María \\ Terapeuta Ocupacional. Master en Gerontología. Profesora Universidad del Rosario. \\ Bogotá. Colombia \\ Contacto > > E mail: anmonsal@urosario.edu.co Transversal 20 No 107-24 apto \\ 401 Teléfono 3474570 ext. 410 \\ Referencia > > Monsalve Robayo, Angélica María. "El Uso productivo del Tiempo \\ Libre vivenciado desde la Animación Sociocultural con personas mayores". Revista \\ Chilena de Terapia Ocupacional. №5. Noviembre 2005.
}

\section{- Abstract}

This article shows the importance of planning and presenting strategies based on designed programs of social and cultural animation that produce continuity, sense of ownership and selfworth to help elderly people take advantage of their leisure time. To start with those programs the Occupational Therapist has to use education, culture, social community work and productive leisure as fundamental tools to support the activity for all of the older population who stay at home. The entitlement and participation on these programs depends on the participant's motivation and the Therapist's creativity and dynamism able to produce interest and taste for the different activities. The related programs are very useful to structure better habits, involve the older participants in leading the groups that they are working in, and make stronger their relationship with people of different ages at the same time that the older person will become more technically aware by working with younger people and by using the new technology and industrialization.

\section{Resumen}

Este artículo muestra la importancia de plantear estrategias para el uso productivo del tiempo libre de personas mayores institucionalizadas a través del diseño de programas de animación sociocultural que generen continuidad, sentido de pertenencia y autogestión. La metodología empleada para su puesta en marcha incluye la aplicación inicial de una lista de chequeo de intereses en donde se describen actividades educativas, culturales, de participación socio comunitaria y de ocio productivo. La clasificación, proposición y ejecución de las actividades de mayor interés escogidas por la población deben planearse teniendo en cuenta variables tales como el género, habilidades, nivel educativo y tiempo estimado de ejecución. La vinculación y participación de las personas mayores dependen permanentemente de la motivación de los participantes y de la creatividad y dinamismo del terapeuta para despertar el interés y placer de la población hacia las actividades. El impacto de los programas de animación sociocultural se evidencia a partir de la estructuración de hábitos, de la motivación de las personas mayores por liderar procesos al interior del grupo, del fortalecimiento de las relaciones intergeneracionales y del contacto directo con la tecnología y el entorno. 


\section{" Introducción "}

El artículo que aquí se presenta surge de la necesidad de conceptualizar y profundizar sobre el uso productivo del tiempo libre como eje fundamental en el estudio de la Ocupación Humana.

Es así como se hace una revisión teórica sobre las diferentes concepciones de tiempo libre y de la animación socio cultural como dimensión interdisciplinar que involucra elementos referidos al entorno en donde personas y comunidades buscan integrarse en un tiempo y espacio determinado, reconociéndose con una nueva actitud ante la vida, un nuevo estilo de creatividad y una nueva posibilidad de relacionarse. ${ }^{(4)}$

Por tanto, el vivenciar estos conceptos desde la cotidianeidad de una población como los adultos mayores constituye uno de los propósitos del trabajo del Terapeuta Ocupacional al buscar responder los siguientes interrogantes:

- ¿Qué intereses ocupacionales se evidencian en los adultos mayores Institucionalizados?

- ¿Cómo la participación en actividades de animación sociocultural favorecen el desempeño ocupacional de los adultos mayores?

- ¿Qué relación existe entre la utilización "productiva del tiempo libre" y la animación socio cultural?

Estas preguntas, entre otras, constituyen el punto de partida en la búsqueda y fundamentación de experiencias y metodologías que llevan consigo un análisis de los enfoques terapéuticos tradicionales utilizados por el Terapeuta Ocupacional en entornos gerontológicos.

Estos enfoques buscaban en primera instancia conservar y mantener una serie de habilidades de ejecución para la realización independiente de actividades básicas cotidianas.

Posteriormente surgen metodologías como los programas de vida independiente y los grupos de adultos mayores que se vinculan a actividades educativas y de voluntariado, los cuales constituyen un primer espacio de participación en donde el objetivo principal no sólo responde a la conservación de habilidades sino a la posibilidad de ser reconocido como parte activa de una sociedad.

En este sentido la importancia que representa el tiempo libre en la vida de las personas es fundamental para satisfacer necesidades de tipo axiológico como el ser, el tener, el hacer y el estar, y de tipo existencial tales como el afecto, el entendimiento, la participación, el ocio, la creación, la identidad y la libertad, según lo propuesto por Max Neef en 1986.

Por tal razón se revisarán aspectos fundamentales y valiosos del tiempo libre como proceso ocupacional.

\section{" Tiempo libre, Tiempo de ocio, Tiempo de productividad "}

Históricamente en Terapia Ocupacional el tiempo libre ha sido considerado como uno de los elementos fundamentales en el estudio de la ocupación humana.

En la terminología uniforme el término "leisure" es analizado como un área del desempeño y definido 
como aquel tiempo que se ocupa en actividades no obligatorias que son motivadas intrínsecamente y que no están relacionadas con actividades de autocuidado, trabajo o sueño (Parham y Fazio, 1997).

Es así como se clasifica en:

1. TIEMPO LIBRE DE EXPLORACIÓN: como su nombre lo indica, se realiza una exploración de intereses, habilidades, oportunidades y de actividades apropiadas de tiempo libre.

2. TIEMPO LIBRE DEDICADO A LA PARTICIPACIÓN: en el cual se incluye la planeación y participación en las actividades apropiadas de tiempo libre; el mantenimiento del equilibrio entre estas actividades y las otras áreas de la ocupación; la obtención, uso y mantenimiento del equipo y ayudas apropiadas para la ejecución de las mismas. ${ }^{(5)}$

A lo largo de la historia, el tiempo libre se ha usado realizando diferentes tipos de actividades, desde la caza y la pesca hasta el uso de la tecnología. En este sentido el adulto mayor se ve enfrentado a nuevos retos y desafíos por lo cual la sociedad actual debe generar cambios actitudinales y culturales, en donde el acceso a programas de bienestar social faciliten la participación de todos los grupos de edad y garanticen la promoción de hábitos saludables.

Joffre Dumazedier, sociólogo francés define el tiempo libre como "la actividad aparte de las obligaciones del trabajo, la familia y la sociedad, en la cual el individuo se vuelca hacia la relajación, la diversión, la ampliación de su conocimiento y hacia la participación social espontánea y del libre ejercicio de su capacidad creativa". ${ }^{(5)}$

Articulado con esto, podríamos afirmar que después de haber ejecutado roles de estudiante, trabajador, padre, hermano, compañero, amigo, abuelo, entre otros, el adulto mayor continúa con la necesidad de ocupar un espacio significativo que le genere satisfacción y en donde pueda sentirse productivo sin ser discriminado por la lentitud de sus movimientos y de su capacidad cognitiva.

En este sentido para Mosey ${ }^{(6)}$, la participación social constituye un factor preponderante en el estudio de los procesos ocupacionales ya que la concibe como las actividades asociadas a patrones organizados de comportamiento que se esperan de un individuo o de la interacción de éste con otros sujetos y que ocurre al interior de un sistema social.

Adicionalmente surge el concepto de ocio, el cual ha sido definido "como toda actividad formativa realizada dentro del tiempo libre" (1) y el cual dentro de los programas dirigidos al adulto mayor ha cobrado importancia, puesto que la decisión libre, autónoma y creativa para el disfrute y satisfacción de necesidades personales y colectivas son su principal fundamento.

\section{Animación Sociocultural "}

El término animación aparece en el siglo XIV. Animación o animar provienen de los términos latinos "animus, ánima, animatis, animare". Cada uno de los términos hace referencia al espíritu, al alma, a la vida, términos simbólicos unidos a la existencia del ser humano. (2)

La animación bajo los conceptos de dinamismo, actividad, intensidad, fuerza, surge en Francia en los años setenta pasando por un concepto netamente lúdico a una definición que abarca las relaciones de las personas con la vida cotidiana.

Es así como toma valor y significado el acompañamiento centrado en los deseos e intereses de las personas y no tanto en la programación de actividades sin sentido. 
En este aspecto, vale la pena resaltar que el tiempo libre se redimensiona hacia la valoración del tiempo como factor esencial en la vida de los seres humanos, en donde la oportunidad y posibilidad de diseñar, planear, desarrollar y ejecutar programas encaminados a la animación sociocultural constituye una necesidad inminente en la prestación de los servicios por parte de los terapeutas ocupacionales hacia la población adulta mayor, ya que proporciona no sólo la satisfacción de necesidades de participación, sino la posibilidad de sentirse valorado en un contexto actual que dirige su atención a los productos más que a los procesos.

Cuando hablamos de adultos mayores hablamos de personas enfrentadas a un cambio de roles y hábitos, donde el tiempo libre cobra protagonismo al ser utilizado de manera productiva en actividades que generen ganancias para la trascendencia como ser humano, que promuevan la integración social, la comunicación intergeneracional y el acceso a los nuevos avances de la ciencia y la tecnología, alcanzando el máximo crecimiento personal y equilibrio ocupacional, respondiendo así a las demandas del entorno.

Elizasu, $2000^{(3)}$ define la animación socio- cultural como un método de promoción comunitaria para estimular el desarrollo cultural de los individuos y de los grupos, procurando sensibilizarles de sus necesidades y aspiraciones, permitiéndoles comunicarse entre sí para valorar sus capacidades creadoras.

Por tanto, el uso productivo del tiempo libre en programas de animación socio- cultural para personas mayores constituye una estrategia de análisis y de aplicación para el terapeuta ocupacional que fundamentalmente busca:

- Generar espacios de participación colectiva en donde el adulto mayor socialice sus intereses y expectativas frente a las actividades propuestas.

- Tomar conciencia de las propias necesidades e identificar las soluciones para satisfacerlas.

- Posibilitar la movilización de líderes entre el grupo de personas mayores que logren empoderarse de su proceso de envejecimiento como posibilidad de crecimiento personal.

- Acceder a la cultura mediante la planeación, organización, búsqueda de información y toma de decisiones para una mejor estructuración de hábitos y un cambio de concepción sobre el envejecimiento, permitiendo crear agentes transformadores de los paradigmas creados sobre la vejez.

- Favorecer el intercambio generacional y el acceso a la tecnología como proceso vital en la adquisición de nuevo conocimiento.

\section{- Una propuesta en permanente construcción "}

Se presenta a continuación un esquema de programa de animación socio-cultural que busca contribuir a la organización, planeación y ejecución de nuevas estrategias de participación social utilizadas por el terapeuta ocupacional, y el cual surge de las experiencias vivenciadas en el ejercicio profesional con población adulta mayor: (figura 1) 


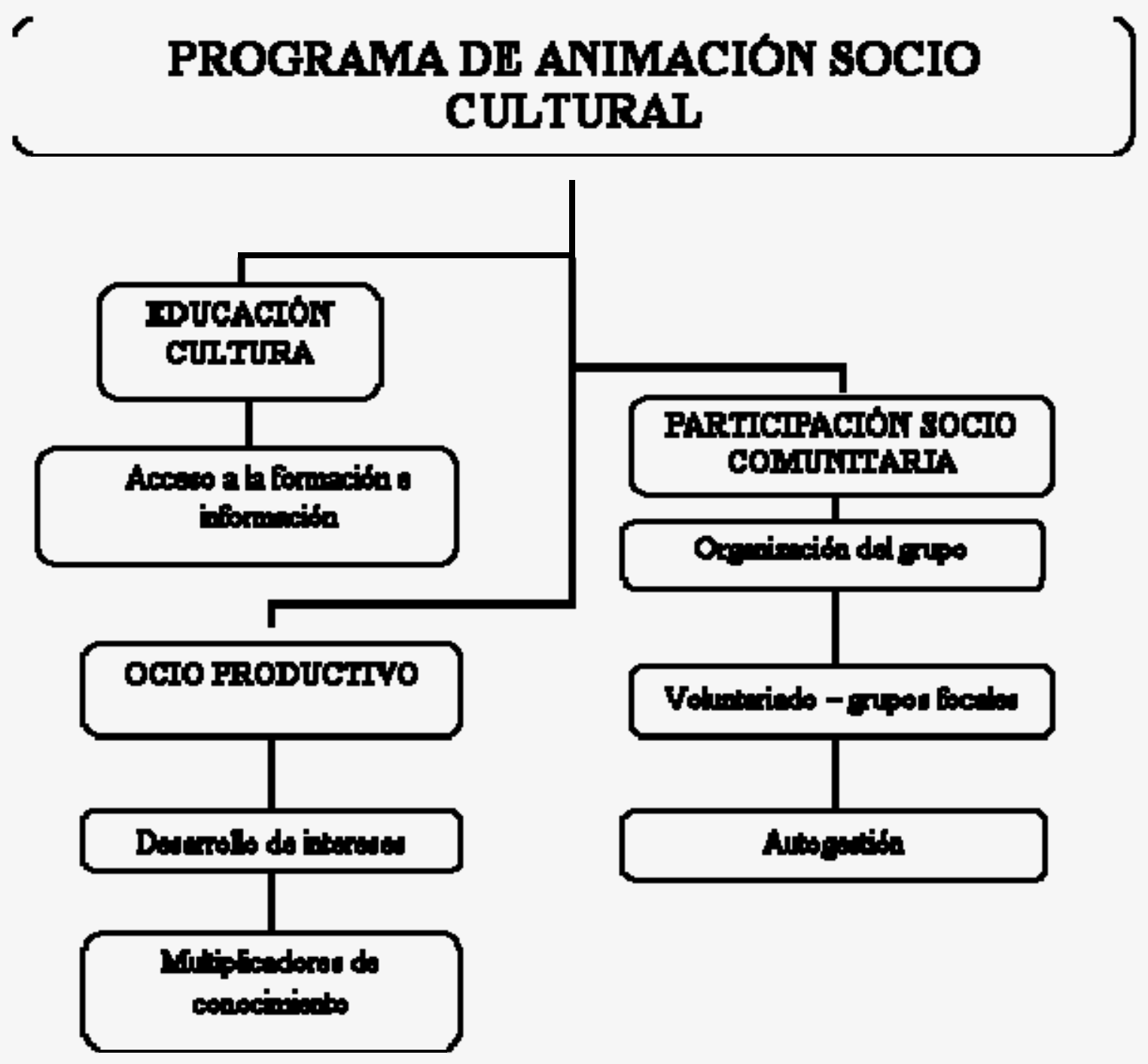

En el esquema se plantean tres componentes fundamentales para el desarrollo de un programa de animación socio-cultural, a saber: educación y cultura; ocio productivo; participación socio comunitaria.

El primero hace referencia a la educación y la cultura como factores inherentes al ciclo vital y a la condición de ciudadano que puede integrarse a procesos de formación y acceder a la información de su cultura, su idiosincrasia, sus intereses, sus posibilidades de participación, a la oferta de servicios de salud, ocio, educación, trabajo, entre otros.

El segundo responde a la necesidad de sentirse productivo a través de la búsqueda y realización de actividades motivantes y autodirigidas que no requieren remuneración económica, pero si gratificación personal y social, en donde el desarrollo de intereses constituyen la base para ser multiplicadores de conocimiento soportado en la historia ocupacional y replicado entre pares y poblaciones de otras generaciones. 
El tercer componente busca constituirse en una alternativa de vinculación fundamentada en la organización de grupos focales de adultos mayores, en donde la capacidad de autogestión y empoderamiento de las acciones planeadas y del análisis de las problemáticas observadas en el contexto inmediato constituyen la razón de ser para participar en dinámicas sociales tales como el voluntariado.

\section{" I mplementación y resultados "}

La implementación del programa que contiene los componentes anteriores se reflejan en la experiencia que a nivel institucional se realizó en un hogar gerontológico de Bogotá que alberga aproximadamente 80 personas mayores de 65 años sin dificultad aparente en la funcionalidad para ejecutar sus actividades de la vida diaria.

La presentación de las diversas actividades del programa incluía el desarrollo de la expresión, la participación cultural y artística, una vertiente comunitaria y asistencial, la motivación para la formación permanente, la dinamización de recursos y la educación en el tiempo libre.

Participaron en total 43 personas de manera voluntaria destacando como principales resultados cualitativos los siguientes:

1. Se desarrolló la función adaptativa e integradora que ayudó a que las personas mayores afrontaran cambios y pérdidas naturales del envejecimiento.

2. Se estimularon las habilidades lúdico-recreativas fomentando el uso y disfrute activo del tiempo libre como medio de diversión, posibilidad de ocuparse y desarrollarse individual y socialmente.

3. Se promovió la capacidad relacional favoreciendo la comunicación, convivencia y establecimiento de relaciones interpersonales.

4. Se desarrolló el espíritu crítico y la capacidad de análisis de la realidad para saber comprender y situar los acontecimientos y fenómenos actuales de nuestra sociedad.

5. Se estimuló la competencia creativa orientada a recuperar y desarrollar potencialidades expresivas de cada persona a través de técnicas como la música, el teatro, el cine, etc.)

6. Finalmente, se fortaleció la capacidad formativa, articulando y reforzando procesos de motivación para el aprendizaje, la recuperación de vivencias, el entrenamiento y mantenimiento intelectual y la actualización de conocimiento adquirido.

Estos resultados surgen de los encuentros realizados con el grupo de personas mayores y la socialización de sus percepciones en relación con las actividades desarrolladas, evidenciando actitudes de participación, dinamismo, cooperación y disponibilidad permanente.

\section{- Conclusión "}

Generar programas de animación socio-cultural es una responsabilidad para quienes trabajamos día a día por el bienestar ocupacional de los adultos mayores. En este sentido las acciones que fundamentan este tipo de programas merecen el análisis y el conocimiento de las posibilidades de participación y de los servicios ofrecidos por entidades públicas y privadas. El uso productivo del tiempo libre en cualquier etapa del ciclo vital es y seguirá siendo objeto de estudio de la Terapia Ocupacional, sin embargo en la población adulta mayor es una necesidad inminente conceptualizar en torno a su significado y a lo que este representa en la vida cotidiana de estas personas, reconociendo la importancia de reflexionar minuciosamente sobre los intereses, valores, contextos, materiales, historia ocupacional, hábitos, motivaciones, como factores que garantizan la realización exitosa del programa a desarrollar. 


\section{" Referencias "}

(1) ELIZASU, C. La animación con personas mayores. Editorial CCS. Madrid. 1999. Pág. $12-13$ y $54-55$

(2) ELIZASU, C. Animación estimulativa para personas mayores. Narcea de Ediciones. Madrid. 2000

(3) GUTIERREZ, L. Métodos para la animación socio-cultural. Editorial CCS. Madrid. 1999

(4) JONES, D, SHEENA, E, HARTERY T, et al. Sociology and Occupational Therapy. Leisure. Chapter 14. Churchill Livingston. 1998

(5) MOSEY, A. Social Participation. 1996, p 340; In: YOUNGSTROM, Mary. The Occupational Therapy Practice Framework: The evolution of Our Professional Language. AJOT. November - December 2002; Volume 56. Number 6: 621

(6) YOUNGSTROM, Mary. The Occupational Therapy Practice Framework: The evolution of Our Professional Language. AJOT. November - December 2002; Volume 56. Number $6: 607-629$ 